\title{
PANGU: A High Resolution Gamma-Ray Space Telescope
}

\author{
X. Wu ${ }^{1 *}$, J. Chang ${ }^{2}$, R. Walter ${ }^{3}$, M. Su ${ }^{4}$, G. Ambrosi ${ }^{5}$, M. Böttcher ${ }^{6}$, M. Chernyakova $^{7}$, \\ Y. Fan ${ }^{2}$, C. Farnier ${ }^{8}$, F. Gargano ${ }^{9}$, I. Grenier ${ }^{10}$, W. Hajdas ${ }^{11}$, M.N. Mazziotta ${ }^{9}$, A. \\ Morselli ${ }^{12}$, M. Pearce ${ }^{13}$, M. Pohl ${ }^{1}$, P. Von Ballmoos ${ }^{14}$, A. Zdziarski ${ }^{15}$, for the PANGU \\ Collaboration
}

${ }^{1}$ Département de Physique Nucléaire et Corpulsculaire, University of Geneva, Switzerland; ${ }^{2}$ Purple Mountain Observatory, Nanjing, China, ${ }^{3}$ INTEGRAL Science Data Center, Department of Astronomy, University of Geneva, Switzerland; ${ }^{4}$ Kavli Institute for Astrophysics \& Space Research, Massachusetts Institute of Technology, Cambridge, U.S.A, ${ }^{5}$ INFN Perugia, Italy; ${ }^{6}$ North-West University, Potchefstroom, South Africa; ${ }^{7}$ Dublin City University, Ireland; ${ }^{8}$ Oskar Klein Center, University of Stockholm, Sweden; ${ }^{9}$ INFN Bari, Italy; ${ }^{10} I R F U$, CEA-Saclay, France; ${ }^{11}$ Paul Scherrer Institute, Villigen, Switzerland; ${ }^{12} I N F N$ Rome, Italy; ${ }^{13}$ Oskar Klein Center, KTH Royal Institute of Technology, Sweden; ${ }^{14}$ IRAP, Toulouse, France; ${ }^{15}$ Nicolaus Copernicus Astronomical Center, Poland.

PANGU (the PAir-productioN Gamma-ray Unit) is a small astrophysics mission with wide field of view optimized for spectro-imaging, timing and polarisation studies. It will map the gammaray sky from $10 \mathrm{MeV}$ to a few $\mathrm{GeV}$ with unprecedented spatial resolution. This window on the Universe is unique to detect photons emitted directly by relativistic particles, via the decay of neutral pions, or the annihilation or decay light from anti-matter and the putative light dark matter candidates. A wealth of questions can be probed among the most important themes of modern physics and astrophysics.

The PANGU instrument is a pair-conversion gamma-ray telescope based on an innovative design of a silicon strip tracker. It is light, compact and accurate. It consists of 100 layers of silicon micro-strip detector of $40 \times 40 \mathrm{~cm} 2$ in area, stacked to height of about $90 \mathrm{~cm}$, and covered by a top anticoincidence detector. PANGU relies on multiple scattering effects for energy measurement, reaching an energy resolution between $30-50 \%$ for $10 \mathrm{MeV}-1 \mathrm{GeV}$. The novel tracker will allow the first polarisation measurement and provide the best angular resolution ever obtained in the soft gamma ray and $\mathrm{GeV}$ band.

PANGU has been proposed to the recent ESA-CAS Call for Joint Small Science Mission. In this contribution, the key science objectives, the payload concept and the expected performance will be presented.

XI Multifrequency Behaviour of High Energy Cosmic Sources Workshop 25-30 May 2015

Palermo, Italy

"Speaker 


\section{Introduction}

We propose a high angular resolution telescope with polarization detection capability dedicated to the sub-GeV $(\sim 10 \mathrm{MeV}$ to $\sim 1 \mathrm{GeV})$ gamma-ray astronomy as a candidate for a future small mission. This mission, called PANGU (PAir-productioN Gamma-ray Unit), will open up a unique window of electromagnetic spectrum that has never been explored with great precision, and will, for the first time, measure the polarization of cosmic gamma rays in this energy range. With such a mission, for 3 years of observation, we can expect to make big steps towards the resolution of several key questions in astrophysics and cosmology:

1) Origin and acceleration mechanism of cosmic rays. It remains an intriguing question where the cosmic rays that have been discovered more than 100 years ago come from, and how they are accelerated to such high energies. Using gamma rays as probes, PANGU can shed new lights on these questions by measuring the spectrum and polarization of gamma rays in the relevant energy range, with great precision, for several categories of likely cosmic ray sources, in particular the Supernova Remnants (SNR), the Active Galactic Nuclei (AGN) and the Gamma-ray Bursts (GRB).

2) Resolution of the Galactic Center. The Milky Way galactic center has been found to emit intense gammay-rays. However past instruments or those in operation have not been successful in resolving this rather extensive region. With an angular resolution about 5-20 times better that previous missions, PANGU can, for the first time, clearly identify the population of point sources and map the diffused emission in this dense region. A clear mapping of the gamma-ray emission in the Galactic Center has important implication on physics of galaxy formation and evolution, and on cosmology.

3) Gamma-ray full sky survey and fundamental physics. With it large field of view, PANGU can accumulate important full sky survey data while completing its main point observation program. The precise gamma ray spectrum covering large region of the sky measured by PANGU may lead to discoveries of fundamental importance, such as the decay and annihilation of Dark Matter, or the Cosmic Gamma-Ray Background (CGRB) resulting from matter-antimatter annihilation after the Big Bang. With an instrument of unprecedented precision to survey the gamma-ray sky in the $10 \mathrm{MeV}-1 \mathrm{GeV}$ region that is very rich in underlying physical process, it is possible that unexpected discoveries of great importance will be made, even with a small mission.

In this proceeding we present an all-silicon tacker-only option, where the energy measurement is based on Coulomb Multiple Scattering. The alternative option of using a permanent magnet spectrometer has beed presented before [1] and will not be discussed here.

\section{Payload Concept}

PANGU is a gamma-ray space telescope consisting of a high spatial resolution silicon microstrip tracker and anticoincidence detectors that detects gamma rays in $10 \mathrm{MeV}-1 \mathrm{GeV}$ energy range based on the electron-positron pair production mechanism. The gamma-ray angular resolution of PANGU is unprecedented, reaching a Point Spread Function (PSF) of $\sim 1^{\circ}$ at $100 \mathrm{MeV}$, and $\sim 0.1^{\circ}$ at $1 \mathrm{GeV}$. The single-end effective areas for vertically incidence gamma 
rays are about $100 \mathrm{~cm}^{2}$ at $100 \mathrm{MeV}$ and $150 \mathrm{~cm}^{2}$ at $1 \mathrm{GeV}$. Compared to other previously proposed high resolution gamma telescope concepts, PANGU is unique in that it dose not need to have a heavy calorimeter for energy measurement, making it suitable to fit into a small mission.
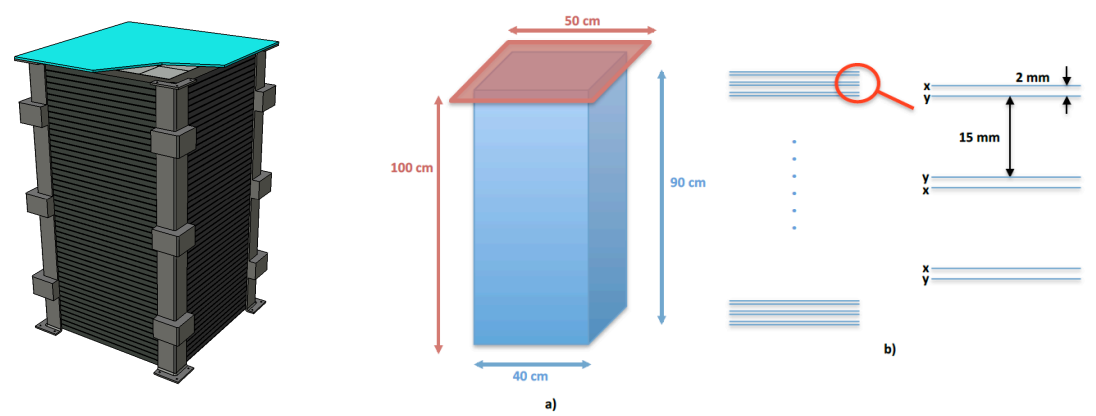

Figure 1: Right: Sketch of the PANGU payload; Left: a) Overall dimensions of the PANGU payload sensitive detectors; $b$ ) Detailed silicon detector layers of the Silicon Tracker

The PANGU payload is approximately a rectangular tower of $50 \times 50 \times 100 \mathrm{~cm}^{3}$, weighs about $60 \mathrm{~kg}$ and consumes about $65 \mathrm{~W}$ of power. The instrument is a high precision tracker make of 100 layers of silicon micro-strip detector of $40 \times 40 \mathrm{~cm}^{2}$ in area, stacked to height of about 90 $\mathrm{cm}$. The tracker is covered on the top end by a plastic scintillator anticoincidence detector. Figure 1 shows a sketch of the PANGU payload and its overall dimensions.

The PANGU silicon tracker employs the robust single-sided strip detector (SSSD) sensor technology already used in previous gamma-ray missions (AGILE, FERMI). The important improvement on the PSF comes from the fact that no passive photon converter material will be used in PANGU, and the thickness of the silicon detector is reduced to as thin as practically possible $(150 \mu \mathrm{m})$, thus minimizing degradation of the angular resolution due to the Multiple Coulomb Scattering (MSC), which is the dominant contribution to the resolution at the energy range of $10 \mathrm{MeV}$ to $1 \mathrm{GeV}$.

To compensate the loss of conversion efficiency due to the thin sensor, 100 layers of silicon detectors are used, organized as $50 \mathrm{x}-\mathrm{y}$ planes of double layers. Each plane has two orthogonal detection layers, one to measure the $\mathrm{X}$ coordinate, another the $\mathrm{Y}$ coordinate, with a distance of $2 \mathrm{~mm}$. The inter-plane distance is $1.5 \mathrm{~cm}$, as shown in Figure 1.

To characterize a gamma-ray source it is necessary to measure the energy of the photon to better than $50 \%$. Measuring the energy of the electron and positron separately helps also to improve the PSF since energy weighted average of the measured directions of the two particles provides a better reconstruction of photon direction than just using the bisector. In previous missions the energy measurement is done with a calorimeter of dense material to force the electron and the positron to deposit all their energies in it. For a $60 \mathrm{~kg}$ payload the use of a calorimeter is practically impossible because of its weight. For PANGU we have developed a concept to measure the energy of the electron and positron using the tracker itself through the measurements of the Multiple Coulomb Scattering (MSC) angles of these particles as they pass through the detector. MSC is the process in which the trajectory of a charged particle traversing a medium is randomly deviated by its interaction with the Coulomb field of the nuclei. The distribution of the scattering angle follows approximately a Gaussian distribution around the original direction, with a standard deviation inversely proportional to the momentum of the 
charged particle, for a given amount material traversed. This is the so-called Highland formula:

$$
\sigma_{\theta} \equiv \theta_{M C S}=\frac{13.6}{\beta p} z \sqrt{\frac{x}{x_{0}}}\left[1+0.038 \ln \left(\frac{x}{x_{0}}\right)\right], \text { where } p \text { is the momentum of the charged particle in }
$$

$\mathrm{MeV}, z$ the charge of the particle, $x$ the distance traversed and $X_{0}$ the radiation length of the medium. It is then possible to measure the momentum of the particle if the scattering angle can be sampled many times in order to extra $\theta_{\text {MCS }}$. The method of measuring momentum with MSC angles has been successfully applied before, for example with nuclear emulsion chambers [2] and large drift chambers [3], and can be used with a silicon tracker as well. However the MSC method requires a better resolution of the track angle measurement than what is needed if only the photon PSF is concerned. The track angle resolution depends mainly on two factors: the position resolution of each measurement point and the distance (lever arm) between two measurement points: $\sigma_{\theta, \text { track }}=\sqrt{2} \sigma_{x} / d$, where $\sigma_{\mathrm{x}}$ is the position resolution and $d$ the lever arm. To improve the position resolution the PANGU SSSD sensor will have a physical pitch of $50 \mu \mathrm{m}$ and a readout pitch of $100 \mu \mathrm{m}$, which together with the analogue readout scheme, provides a $\sigma_{\mathrm{x}}$ of $\sim 17 \mu \mathrm{m}$. As shown in Figure 1 the distance between two measurement points of the same coordinate is $1.5 \mathrm{~cm}$, leading to a basic track angular resolution of $\sim \sqrt{2} \times 0.017 / 15=1.6$ mrad. With this layout, preliminary Monte Carlo simulation (see next section) shows that in the energy range of PANGU $(10 \mathrm{MeV}-1 \mathrm{GeV})$, it is possible to achieve an energy resolution of better than $50 \%$ for gamma rays.

\section{Performance Assessment}

To assess the performance of the payload, in particular in terms of effective area, point spread function and energy resolution, the PANGU payload has been simulated with full details using the GEANT4 [4] program. Here some preliminary results using ideal conditions (for example support structure, electronic noise and trigger dead time are not included) are presented.

\subsection{Effective Area and Acceptance}

Figure 2 left panel shows the effective area (approximately the geometrical area times the photon conversion probability) at different incidence angles: normal incidence (defined as $\cos \theta>0.975), 45^{\circ}$ and $60^{\circ}$. Above $100 \mathrm{MeV}$, the on-axis and off-axis Effective Areas are comparable. Below $100 \mathrm{MeV}$, the off-axis Effective Area is smaller because of the larger pair open angle, which implies more frequent losses of one of the inclined tracks. Figure 2 right panel compares the effective area for $320 \mu \mathrm{m}$ thick silicon detector to that of the default thickness $(150 \mu \mathrm{m})$ at normal incidence. Th effective area is increased with thicker detectors but the angular resolution is degraded (see Section 3.2).

The acceptance and as well as the field of view, defined as the acceptance divided by the on-axis effective area, as function of the gamma-ray energy are shown in the left and tight panel of the Figure 3, respectively. The Field of View is around 1 steradian, corresponding to an observation cone of $33^{\circ}$ half-angle. This means that PANGU has a rather large field of view, at about $60^{\circ}$. 

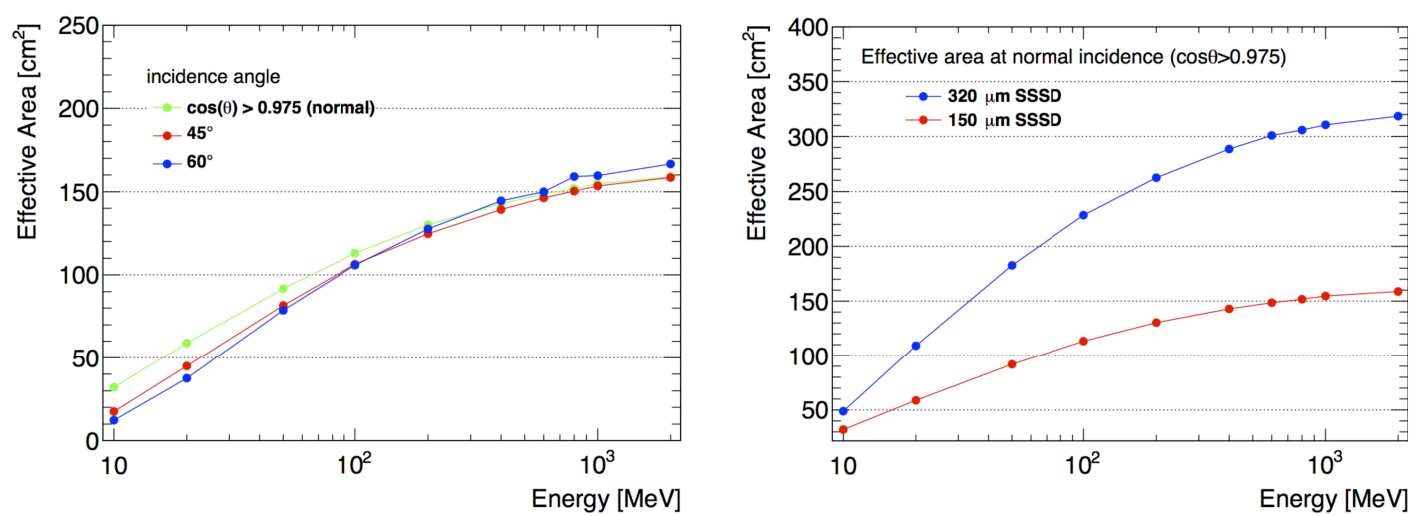

Figure 2: Effective area as function of the gamma-ray energy, for different incidence angles (left); Effective area as function of energy at normal incidence for 2 silicon detector thickness(right).
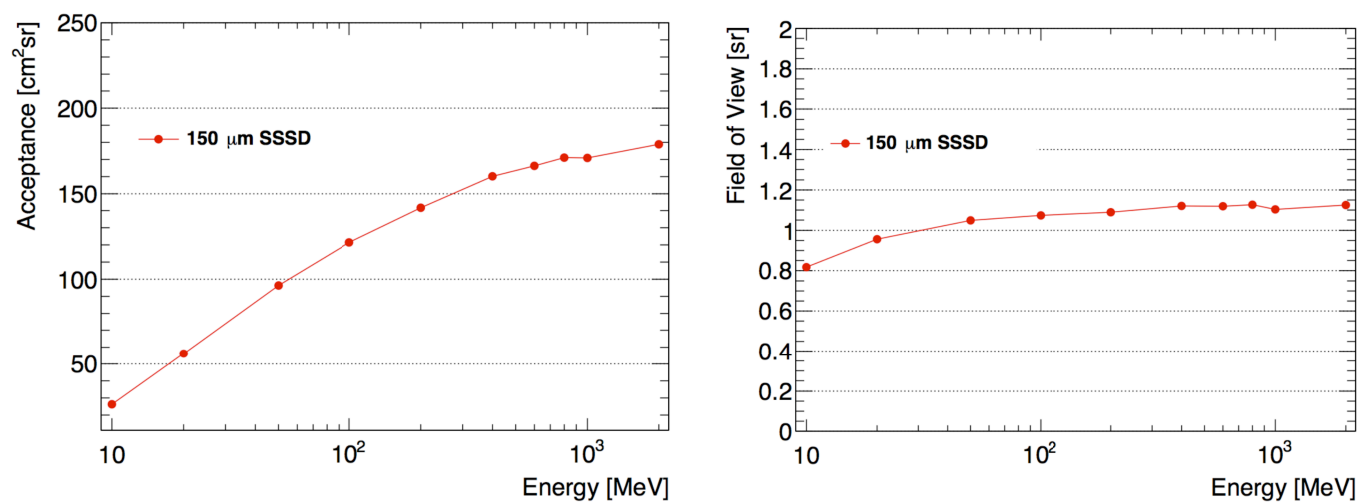

Figure 3: Acceptance (left) and Field of View (right) as function of the gamma-ray energy,

\subsection{Angular Resolution}

Figure 4 (left panel) shows the $68 \%$ and $95 \%$ containment Point Spread Function (PSF) of PANGU at normal incidence, for the default silicon detector thickness $(150 \mu \mathrm{m})$ and a thicker one $(320 \mu \mathrm{m})$. The angular resolution is around $1^{\circ}$ at $100 \mathrm{MeV}$, about 5 times better than Fermi. The degradation of PSF by doubling the silicon detector thickness is $\sim 30 \%$, which can also be used as an indication of degradation due to supporting material. Also note that the ratio of $95 \%$ to $68 \%$ PSF is about 2 across the full energy range, corresponds well to what is expected of a Gaussian distribution. This means that there is very small non-Gaussian tail in the resolution across the full energy range. This impressive feature of PANGU comes from the fact that the detector layers are very thin and there is no calorimeter, so there is very little bremsstrahlung or back scattering effects that could generate tails in the distribution.

The off-axis PSF of PANGU is still excellent, only worsen by $\sim 10(20) \%$ at incidence angle of $45^{\circ}\left(65^{\circ}\right)$, compared to normal incidence, as shown in the right panel of Figure 4. 

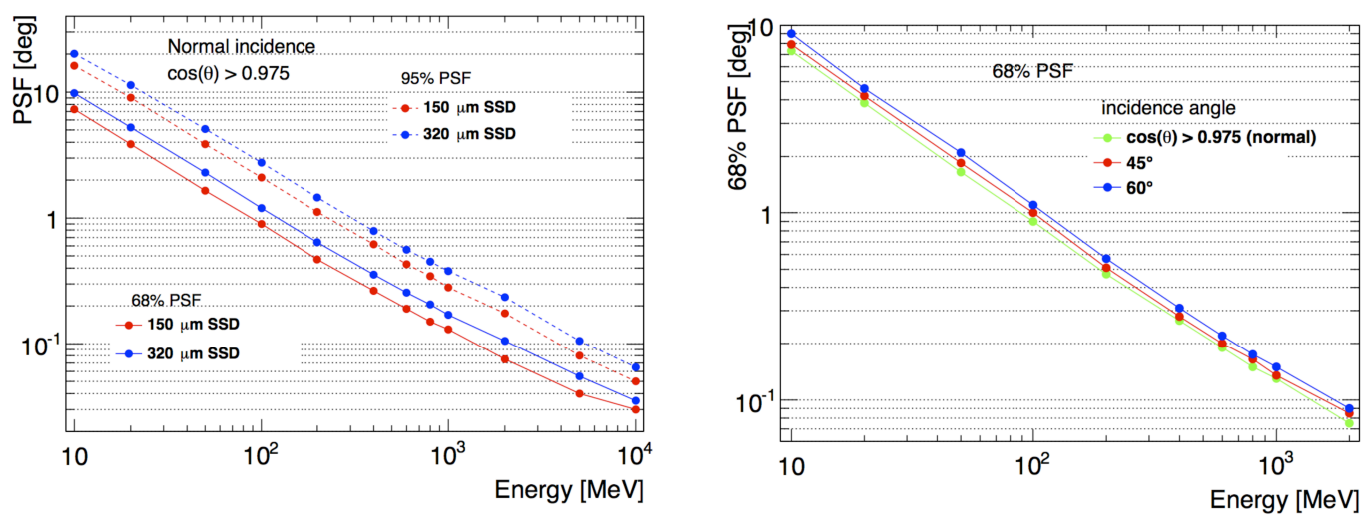

Figure 4: Acceptance (left) and Field of View (right) as function of the gamma-ray energy,

\subsection{Energy Resolution}

As discussed in Chapter 2, the momentum of the electron and the positron can be estimated by measuring the standard deviation (or width), $\theta_{M C S}$, of the distribution of the scattering angle. Since the scattering angles are measured by track reconstruction, the width of the measured scattering angles contains also a contribution due to the uncertainty of track reconstruction, thus: $\sigma_{\text {mes }}^{2}=\sigma_{M C S}^{2}+\sigma_{\theta, \text { track }}^{2}$. The baseline design of PANGU has a $\sigma_{\theta, \text { track }}$ of 1.6 mrad for the basic "track segment" formed by two points of the same measuring coordinated with $1.5 \mathrm{~cm}$ apart (see Figure 1 right, b). According to the Highland formula, $1.6 \mathrm{mrad}$ corresponds to a $\theta_{M C S}$ generated by a charged track of $550 \mathrm{MeV}$ crossing $600 \mu \mathrm{m}$ of silicon, which is the amount of silicon of 4 layers of detectors between two consecutive track segments in the PANGU tracker. This means that the momentum of a charged particle below $550 \mathrm{MeV}$ can be estimate by measuring the width of distribution of scattering angle between successive track segments ("single-segment scattering angles"). For higher energy, scattering angles between track segments that are more than one segment apart ("multi-segment scattering angles") need to be added.

An optimal statistical method to measure the track momentum with multiple scattering effects will be developed during the study phase. Preliminary assessment using the Multiple Model Adaptive Estimate (MMEA) with Kalman filter (used e.g. in spacecraft navigation and tracking) shows promising results. In this framework $\theta_{M C S}$ represents the "process noise" of a know process (MCS in our case), with an unknown parameter (the momentum). The simplest MMEA method uses multiple Kalman filters in parallel to scan the parameter in order to find the parameter value that fits best with the measurements. The left panel of Figure 5 is the result of the implementation of MMEA for PANGU, showing the momentum resolution for charge particles of several values of momentum between $50 \mathrm{MeV}$ and $1 \mathrm{GeV}$, of normal incidence, using a track length of $30 \mathrm{~cm}$. It can be seen that the momentum resolution obtained is between 30 to $40 \%$. The right panel of Figure 5 is an estimate of the gamma-ray energy resolution using simple Gaussian smearing on the momenta of the electron and the positron, with a width of $40 \%$ for tracks below $1 \mathrm{GeV}$ and $50 \%$ for tracks above. The resulting photon energy resolution is about $40 \%$. In reality the non-Gaussian tails of the momentum resolution, as seen in the distributions of higher energy tracks in the left panel of Figure 5, will degrade somewhat the 
energy resolution, but on the other hand more elaborate statistical methods can also improve the final energy resolution.
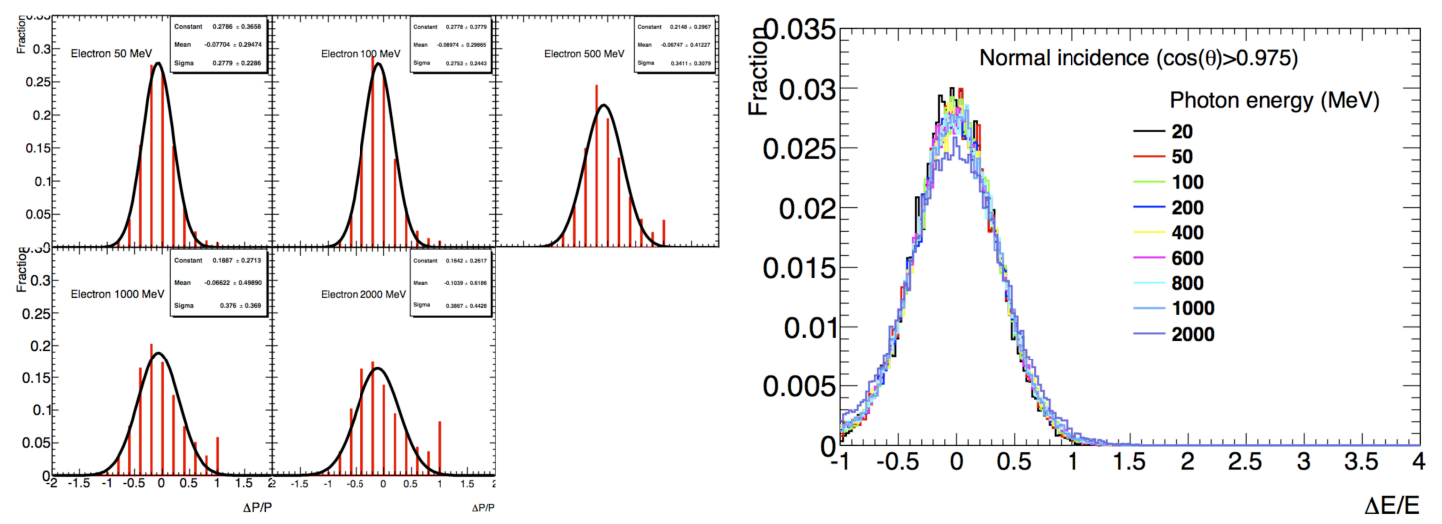

Figure 5: Left) Momentum resolution estimate of normal incidence charge particle using the Kalman filter Multiple Model Adaptive Estimate method; Right) Gamma-ray energy resolution estimated by 40\%$50 \%$ Gaussian smearing of the electron and positron momentum (see text for details).

\subsection{Polarization Detection}

The excellent track angular resolution of PANGU allows to measure the polarization of gamma rays in the pair production regime. A polarized gamma-ray source will generate an asymmetry in the distribution of the azimuthal angle $\phi$ of the electron-positron pair:

$d \sigma / d \varphi=2 \pi \sigma_{0}\left(1+P_{\gamma} A \cos \left(2 \varphi-2 \varphi_{p o l}\right)\right)$, where $P_{\gamma}$ is the polarization of the photon and $A$ is the modulation factor of the detection process. In the case of the pair production, $A$ depends on the energy of the photon as well as the kinematics of the electron and position thus cannot be evaluated analytically. Numerical integration gives a value between 0.1 and 0.5 , with an average about 0.2 . One of the work packages of the proposal is precisely the detailed study of the analysing power of the pair production process, and to validate existing or implement new code for pair production simulation for polarized photons. From the payload point of the view the polarization detection capability can be characterized by the detection of non-zero $P_{\gamma} \cdot \mathrm{A}$ values. Figure 6 shows the azimuthal angular distribution of the leading track for 4 different values of input $\mathrm{P}_{\gamma} \cdot \mathrm{A}$, for $100 \mathrm{MeV}$ and $1 \mathrm{GeV}$ gamma rays, and for 2 polarization angles $\left(0^{\circ}\right.$ and $\left.30^{\circ}\right)$. It can be seen that the intrinsic detector asymmetry (dotted black lines) due to the orientation of the silicon strips is relative small. With sufficient statistics ( $\sim 60 \mathrm{k}$ photons) it is possible to detect down to a $P_{\gamma}: A \sim 10 \%$ up to $1 \mathrm{GeV}$.
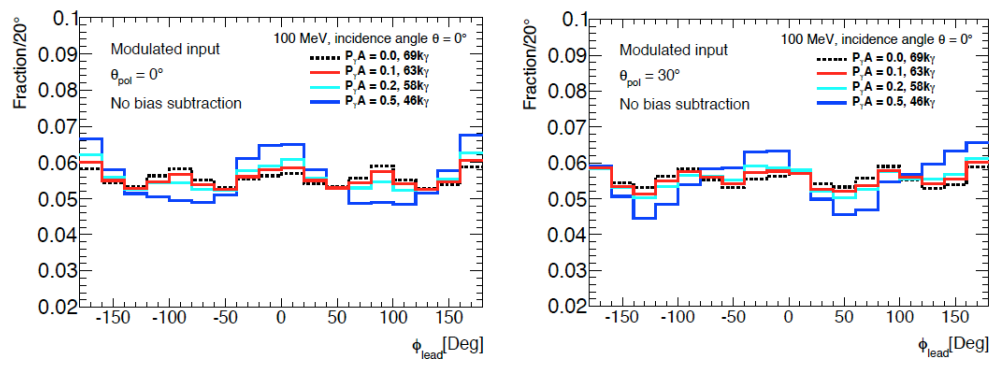

Figure 6: Azimuthal angular distributions of the leading track for 4 different $P_{\gamma} \cdot A$ values: 0 (black dotted lines), 0.1 (red lines), 0.3 (cyan lines) and 0.5 (blue lines), for $100 \mathrm{MeV}$ (two top panels) and 1 $\mathrm{GeV}$ (two bottom panels), and two
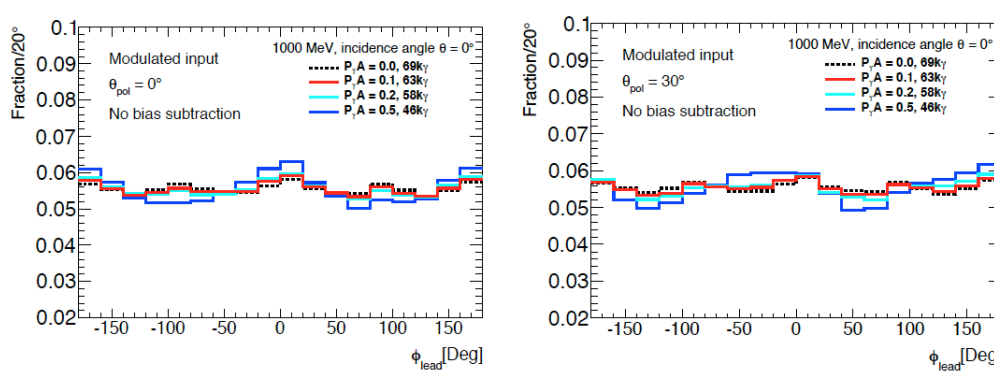
different polarization angle: $0^{\circ}$ (left two panels) and $20^{\circ}$ (right two panels). 


\section{Conclusions}

We present the all-silicon tracker-only payload option for the sub-GeV high resolution gamma-ray space telescope PANGU (PAir-productioN Gamma-ray Unit). The new payload concept is based on the mission-proven silicon micro-strip tracker technology, but with an innovated design to make a simple yet powerful gamma ray detection unit that has the functions of a imager, a spectrometer and a polarimeter all in the same unit that can fit into a small class mission below $100 \mathrm{~kg}$. PANGU will provide the first high resolution full-sky map in the energy range from $\sim 10 \mathrm{MeV}$ to $\sim 1 \mathrm{GeV}$. It will survey the full sky with a size of $68 \%$ containment of the point spread function (PSF) $\leqslant 1^{\circ}$ at $\sim 100 \mathrm{MeV}$. The high quality gamma-ray maps will significantly improve the identification and separation of point sources from extended and complicated diffuse gamma-ray background. It is a first step towards filling the well-known "medium energy gap" in gamma-ray astronomy and will open up the discovery window in the sub-GeV region.

\section{References}

[1] Wu, X., et al., 2014, Proc. SPIE 9144 Space Telescopes and Instrumentation 2014: Ultraviolet to Gamma Ray, 91440F [arXiv:1407.0710].

[2] Agafonova, N., et al., 2012, New Journal of Physics 14, 013026

[3] Ankowski, A., et al., 2006, Eur. Phys. J. C 48, 667

[4] Agostinelli, J., et al., 2003, NIM A 506, 250, Allison, J. et al., 2006, IEEE Transactions on Nuclear Science, 1, 270 